\title{
Long chain lipids facilitate insertion of large nanoparticles into membranes of small unilamellar vesicles
}

\author{
Marzouq Adan ${ }^{1,2,+}$, Morgenstein Lion ${ }^{2,3,+}$, Yudovich, Shimon ${ }^{2,3}$, Atkins Ayelet $^{2}$, Grupi Asaf ${ }^{2,3}$ \\ *, Weiss Shimon $2,3,4,5,{ }^{*}$
}

${ }^{1}$ Department of Chemistry, Bar-Ilan University, Ramat-Gan, 52900, Israel

${ }^{2}$ Institute for Nanotechnology and Advanced Materials, Bar-llan University, Ramat-Gan, 52900, Israel

${ }^{3}$ Department of Physics, Bar-Ilan University, Ramat-Gan, 52900, Israel

${ }^{4}$ Department of Chemistry and Biochemistry, University of California Los Angeles, Los Angeles, CA 90095

${ }^{5}$ California NanoSystems Institute, University of California Los Angeles, Los Angeles, CA 90095

\begin{abstract}
Insertion of hydrophobic nanoparticles into phospholipid bilayers is limited to small particles that can incorporate into the hydrophobic membrane core in between the two lipid leaflets. Incorporation of nanoparticles above this size limit requires development of challenging surface engineering methodologies. In principle, increasing membrane thickness should facilitate incorporation of larger nanoparticles. Here we explore the effect of very long phospholipids (C24:1) in small unilamellar vesicles, on the membrane insertion efficiency of nanoparticles in the range of $5-13 \mathrm{~nm}$ in diameter. To this end, we improved an existing vesicle preparation protocol and utilized cryogenic electron microscopy imaging to examine the mode of interaction and to evaluate the membrane insertion efficiency of membrane-inserted nanoparticles.
\end{abstract}

+These authors contributed equally to this work.

“Corresponding authors: sweiss@chem.ucla.edu; grupia@gmail.com

Keywords: Quantum dots, nanorod, membranes, long chain lipid, SUV, membrane insertion, cryo-EM.

\section{Introduction}

In recent years, synthesis methods for the production of highquality nanoparticles (NP) have greatly improved, allowing for increased control over their size, shape, chemical and physical properties. This, in turn, afforded the fabrications of sophisticated metallic, magnetic, dielectric, and semiconducting heterostructured NPs with desirable photophysical and chemical properties ${ }^{[1]}$. This precise control allows the engineering of excited-state wavefunctions ${ }^{[2]}$, charge confinement, and spatiotemporal control of chargeseparated states ${ }^{[3]}$. Hence, NPs (and in particular semiconducting) have proved to be very useful in diverse applications such as in optoelectronics ${ }^{[4]}$, biological imaging ${ }^{[5]}$, sensing ${ }^{[6]}$, catalysis ${ }^{[7]}$, energy harvesting ${ }^{[8]}$, biomedicine, and cell surface engineering ${ }^{[9]}$.
While these sophisticated inorganic nanomaterials require advanced synthesis methods, their integration with biology macromolecules and machineries requires additional functionalization steps. Current approaches typically target NPs to interact with the cell membrane's surface or to undergo a cellular uptake. Less effort has been invested in functionalization of NPs that could be targeted, incorporated into, and retained in the membrane bilayer core itself. Such membrane inserted NPs could expand the repertoire of desired cellular functions while taking advantage of the superior inorganic materials' properties. ${ }^{[10]}$ Once inserted into the membrane, such NPs could, for example, introduce orthogonal (to native signaling) ways to communicate with the cell's interior, introduce de-novo or enhance native enzymatic/catalytic activities, sense the membrane potential, used as antennas for light harvesting and vision restoration ${ }^{[1 \mathrm{~d}}$, $11]$.

The underlying obstacle for stable insertion of large NPs into lipid membranes is that in many cases, the surfaces of assynthesized NPs are decorated with a mixture of highly hydrophobic ligands and are insoluble in biologically-relevant aqueous media. Therefore, without any surface modification, only small, hydrophobic NPs can be efficiently incorporated into synthetic vesicle membranes in between the two leaflets. Since phospholipid bilayers are typically $4-5 \mathrm{~nm}$ thick ${ }^{[12]}$, successful membrane insertion has been achieved only for NPs with sizes $\leq 5 \mathrm{~nm}^{[13]}$. Inclusion of larger hydrophobic NPs is thermodynamically unfavorable due to the energetic penalty associated with protrusion of hydrophobic ligands into the polar solvent. ${ }^{[13 c]}$ This limitation practically excludes higher order structures and functionalities that could be beneficial in terms of signal or actuation strength.

We hypothesized that increased membrane thickness should promote insertion of larger NPs than previously accomplished. The thickness of phospholipid membrane can be increased by incorporation of lipids with a long alkane chain and by addition of cholesterol which aligns and stretches alkane chains into a packed and thick membrane ${ }^{[12 a]}$. To test this hypothesis, we prepared small unilamellar lipid vesicles incorporating 1,2dinervonoylsn-glycero-3-phosphocholine (PC24), an unsaturated zwitterionic phospholipid with 24 carbon alkane chains. This lipid has been previously used in the preparation of multilamellar liposomes (MLVs) formulations with protein or 
peptide drugs, and in the formation of planar bilayer lipid membranes ${ }^{[14]}$. In biological systems, it is a component of lipid rafts and micro domains ${ }^{[15]}$.

Compared to typical PC18 lipids which form membranes of 4$5 \mathrm{~nm}$ thickness, lipid bilayers consisting of $25 \%$ PC24 are expected to lead to a $20 \%$ increase in membrane thickness, i.e., up to $6 \mathrm{~nm}$. To test our hypothesis, we evaluated the membrane-insertion efficiency of inorganic NPs in the size range of $5-13 \mathrm{~nm}$ in diameter into the lipid bilayer of small unilamellar vesicles (SUVs) containing various molar fractions of PC24 during the formation of lipid vesicles. Initial attempts to incorporate even small NPs into SUVs prepared by the hydration method ${ }^{[16]}$ were unsuccessful. After testing several other SUV preparation protocols for NP insertion that failed, we identified in the literature a protocol that was previously used to encapsulate hydrophobic drugs into liposomes' membranes ${ }^{[17]}$ but never applied for membrane incorporation of nanoparticles. This approach proved to be highly efficient for membrane insertion of small NPs. A critical step in this protocol is the addition of a lipid detergent to the organic solution containing both lipids and NPs. With the addition PC24 to the lipid mixture ${ }^{[14]}$, NPs with diameters of up to $\sim 13 \mathrm{~nm}$ were successfully incorporated into SUVs' membranes.

Here we present a detailed protocol for incorporation of large NPs (up to $\sim 13 \mathrm{~nm}$ ) into the lipid membrane of SUVs with optimized lipid composition, detergent, organic solvents and NP/lipid ratio. Cryogenic electron microscopy (cryo-EM) imaging of these membrane-inserted NPs was used for the characterization of their mode of interaction with the membrane.

\section{Results}

In order to insert NPS efficiently to SUVs, we adopted a detergent dialysis vesicle preparation method that was previously optimized for membrane incorporation of small hydrophobic drugs ${ }^{[17]}$. This protocol was modified here to allow for NPs' membrane insertion. Two different mixtures of lipids were used for SUVs preparations: 'native membrane', with POPC, cholesterol and DOTAP (central column in table 1) and 'thick membrane', with POPC, cholesterol, DOTAP, and a very long-chain phospholipid (PC24). Insertion efficiency was tested for four different sizes of NPs: 5, 7, and $11 \mathrm{~nm}$ spherical QDs, and $5 \times 13 \mathrm{~nm}$ cylindrical-shaped NRs (sizes determined by TEM, Table $\mathrm{S} 1$ ).

Cryo-EM imaging was used to assess the mode of NPs interaction/insertion with/into the SUVs' membrane. As described in the supplementary information section, the yield of QDs / NRs insertion into the membrane was evaluated by examining the cryo-EM images and counting membraneinserted NPs, membrane-adsorbed NPs, and encapsulated NPs. We excluded all NPs that were either interacting with the carbon grid or aggregated. Multiple experiments on different days were conducted for each condition (NP size/type and 'native' or 'thick' membrane). Typically, $>3$ different experiments were conducted for each condition, with several field-of-views (FOVs) on the grid.

In order to find the optimal concentration of PC24 in the lipid mixture, we first tested five preparations of SUVs using different relative concentrations of PC24 and evaluated NRs insertion efficiency as function of [PC24] (Table S2). NRsembedding SUVs were prepared as described in the supplementary information section, with NRs concentrations carefully maintained for all 5 preparations. Each preparation was imaged by cryo-EM and subsequently analyzed to assess the mode of NR-membrane interaction. Figure 1 shows NRs membrane insertion efficiency as a function of the longchained lipid concentration [PC24] in the lipid mixture. The figure shows an optimal concentration of $[P C 24]=25 \%$ of the total lipid molar concentration for which the insertion efficiency is $\sim 25 \%$ (which is twice higher than the insertion efficiency of SUVs prepared with the 'native' membrane (2 out of 7 compared to 5 out of 10). Higher or lower [PC24] showed a decreased insertion efficiency as compared to $[P C 24]=25 \%$. Although [PC24] $=100 \%$ also resulted in good NRs insertion, which was comparable to [PC24] $=25 \%$, the morphology of these SUVs was not spherical (Figure S2). The non-spherical, polygonal SUV shapes can be attributed to the membrane's gel phase behavior, since PC24 has a transition temperature of $26{ }^{\circ} \mathrm{C}^{[18]}$, which is higher compared to the transition temperature of POPC $\left(-2{ }^{\circ} \mathrm{C}\right)$. In addition, at [PC24] $=50 \%$, SUVs formation had remarkably decreased (data not shown). Both of these observations will be further investigated in the future.

Figure 2 shows cryo-EM images of selected examples of successful insertions of the different size/type NPs into 'thick' membranes. The morphology of SUVs at different C24 concentrations is shown in Supplementary Figure S2, and raw images are deposited (10.5281/zenodo.5068489). The insertion efficiency was defined as the ratio between the number of membrane-inserted NPs and the total number of NPs in a frame. Results from all analyzed frames per condition were averaged. The left column of Figure 2 shows representative images of particle insertion. The blue and red bars on the right columns represent the fractions of inserted and non-inserted particles, respectively, out of the entire counted particles, excluding aggregates and carbon interacting particles.

As expected, insertion efficiency was inversely proportional to the NP size for 'native' membranes due to the particle size, whereas the long lipid addition had significant effect on the larger particle as a result of the increase of the membrane thickness. Interestingly, when using $[\mathrm{PC} 24]=25 \%$ ('thick' membrane), the largest size QDs (11 nm) could be inserted into the membrane at $50 \%$ efficiency, while they could not be inserted at all into the 'native' membrane. For other sizes, we saw an increase from $46 \%$ to $70 \%$ for the $5 \mathrm{~nm}$ QDs, $40 \%$ to $48 \%$ for the $7 \mathrm{~nm}$ QDs, and $28.5 \%$ to $50 \%$ for the $5 \times 13 \mathrm{~nm}$ NRs.

We further evaluated the degree of insertion for the $11 \mathrm{~nm}$ QDs by localizing the position of QDs relative to the SUVs' membrane using a home written software, as described in the supplementary information section. Figure $3 d$ shows a histogram of these distances where positive distances represent locations in the outer SUV leaflet and outwards the SUV, and negative distances represent locations in the inner SUV leaflet and inwards the SUV. The histogram shows that these large NPs are probable to be located at the center of the membrane. Figures 3 (a)-(c) show 3 examples of analyzed FOVs; as an example for the analysis.

\section{Discussions}

Previous works have shown that only very small QDs $(<4$ $\mathrm{nm})^{[13]}$ can be incorporated into SUVs' membranes during their formation using electro-swelling, sonication and other 
bioRxiv preprint doi: https://doi.org/10.1101/2021.07.12.452073; this version posted July 13, 2021. The copyright holder for this preprint (which was not certified by peer review) is the author/funder, who has granted bioRxiv a license to display the preprint in perpetuity. It is made available under aCC-BY-NC 4.0 International license.

detergent removal protocols ${ }^{[19]}$. Here, we used a different approach that relied on a previously published protocol for membrane incorporation of small hydrophobic drug molecules. ${ }^{[17]}$ In this approach, a dialysis-based detergent removal step was performed during the preparation of the vesicles. This improved protocol was sufficient for the insertion of larger (> $5 \mathrm{~nm}$ ) particles into SUV membrane composed of POPC and cholesterol, although at low efficiency. The addition of a long-chain phospholipid, PC24, significantly improved the insertion of large particles into SUV membranes, and facilitated the insertion of large QDs $(\sim 11 \mathrm{~nm})$ at high efficiency. Combining both approaches, NPs as large as $11 \mathrm{~nm}$ QDs and $5 \times 13 \mathrm{~nm}$ NRs were successfully inserted into SUVs.

It is well accepted that membrane thickness varies with acyl chain length and the presence of cholesterol. We found that adding long chain phospholipids (PC 24:1) to the vesicle formulation is crucial for the incorporation of NPs with diameters much larger than those reported for typical lipid compositions. Although the main obstacle for NPs incorporation into SUVs is their size; the effect of the hydrophobic capping ligand layer and the particle geometry on its insertion into SUVs is a matter of future study.

\section{Summary}

In summary, we developed a lipid composition and SUV preparation protocol that promotes integration of large inorganic hydrophobic particles into the membrane. These SUVs could be made fusogenic, to allow the delivery of NPs into cellular membranes. The use of long-chain lipids together with the addition of the detergent in the organic phase are crucial for successful membrane insertion of large NPs. The technology developed here could be applied to diagnostic technologies, therapeutic applications, and as a research tool for studying membrane properties.

\section{Acknowledgements}

We thank Prof. Dan Oron and Dr. Gaoling Yang for providing the NR sample. This work has received funding from the European Research Council (ERC) under the European Union's Horizon 2020 research and innovation program under grant agreement No. 669941 and ERC-POC grant agreement No. 779896, by the BER program of the Department of Energy Office of Science grant DE-SC0020338, by the STROBE National Science Foundation Science \& Technology Center, Grant No. DMR-1548924, by the Israel Science Foundation grant \# 813/19, and by the Bar-llan Research \& Development Co, the Israel Innovation Authority, Grant No. 63392. 


\section{References}

[1] aZ. A. Peng, X. Peng, Journal of the American Chemical Society 2002, 124, 3343-3353; bX. Peng, L. Manna, W. Yang, J. Wickham, E. Scher, A. Kadavanich, A. P. Alivisatos, Nature 2000, 404, 59-61; cL. Manna, E. C. Scher, A. P. Alivisatos, Journal of the American Chemical Society 2000, 122, 12700-12706; dV. De Leo, L. Catucci, A. Falqui, R. Marotta, M. Striccoli, A. Agostiano, R. Comparelli, F. Milano, Langmuir 2014, 30, 1599-1608.

[2] aN. N. Hewa-Kasakarage, M. Kirsanova, A. Nemchinov, N. Schmall, P. Z. El-Khoury, A. N. Tarnovsky, M. Zamkov, Journal of the American Chemical Society 2009, 131, 1328-1334; bJ. Müller, J. M. Lupton, A. L. Rogach, J. Feldmann, D. V. Talapin, H. Weller, Physical Review B 2005, 72; cD. V. Talapin, J. H. Nelson, E. V. Shevchenko, S. Aloni, B. Sadtler, A. P. Alivisatos, Nano Letters 2007, 7, 2951-2959.

[3] J. Müller, J. M. Lupton, P. G. Lagoudakis, F. Schindler, R. Koeppe, A. L. Rogach, J. Feldmann, D. V. Talapin, H. Weller, Nano Letters 2005, 5, 2044-2049.

[4] aT.-H. Kim, K.-S. Cho, E. K. Lee, S. J. Lee, J. Chae, J. W. Kim, D. H. Kim, J.-Y. Kwon, G. Amaratunga, S. Y. Lee, B. L. Choi, Y. Kuk, J. M. Kim, K. Kim, Nature Photonics 2011, 5, 176-182; bV. I. Klimov, S. A. Ivanov, J. Nanda, M. Achermann, I. Bezel, J. A. McGuire, A. Piryatinski, Nature 2007, 447, 441-446.

[5] X. Michalet, F. F. Pinaud, L. A. Bentolila, J. M. Tsay, S. Doose, J. J. Li, G. Sundaresan, A. M. Wu, S. S. Gambhir, S. Weiss, Science 2005, 307, 538.

[6] aS. Li, K. Zhang, J.-M. Yang, L. Lin, H. Yang, Nano Letters 2007, 7, 3102-3105; bM. J. Ruedas-Rama, E. A. H. Hall, Analytical Chemistry 2008, 80, 8260-8268; cH. Aouani, S. Itzhakov, D. Gachet, E. Devaux, T. W. Ebbesen, $\mathrm{H}$. Rigneault, D. Oron, J. Wenger, ACS Nano 2010, 4, 4571-4578.

[7] H. Zhu, N. Song, H. Lv, C. L. Hill, T. Lian, J Am Chem Soc 2012, 134, 11701-11708.

[8] I. Mora-Seró, J. Bisquert, The Journal of Physical Chemistry Letters 2010, 1, 30463052.

[9] aR. F. Fakhrullin, A. I. Zamaleeva, R. T. Minullina, S. A. Konnova, V. N. Paunov, Chem Soc Rev 2012, 41, 4189-4206; bJ. Z. Wang, Z. Q. Ding, F. Zhang, W. B. Ye, Mater Sci Eng C Mater Biol App/ 2017, 77, 12471260; cB. J. Kim, H. Cho, J. H. Park, J. F. Mano, I. S. Choi, Adv Mater 2018, 30, e1706063; dT. Liu, Y. Wang, W. Zhong, B. Li, K. Mequanint, G. Luo, M. Xing, Adv Healthc Mater 2019, 8, e1800939.

[10] aP. Pandey, S. P. Singh, S. K. Arya, V. Gupta, M. Datta, S. Singh, B. D. Malhotra, Langmuir 2007, 23, 3333-3337; bG. Tikhomirov, S. Hoogland, P. E. Lee, A. Fischer, E. H. Sargent, S. O. Kelley, Nat Nanotechnol 2011, 6, 485-490.

[11] aM. E. Åkerman, W. C. W. Chan, P. Laakkonen, S. N. Bhatia, E. Ruoslahti, Proceedings of the National Academy of Sciences 2002, 99, 12617; bA. M. Derfus, W. C. W. Chan, S. N. Bhatia, Advanced Materials 2004, 16, 961-966.

[12] aK. Cathcart, A. Patel, H. Dies, M. C. Rheinstadter, C. Fradin, Membranes (Basel) 2015, 5, 664-684; bS. Tharad, O. Uzulmez, B. Promdonkoy, J. L. Toca-Herrera, Int J Mol Sci 2018, 19.

[13] aV. De Leo, F. Milano, A. Paiano, R. Bramato, L. Giotta, R. Comparelli, S. Ruscigno, A. Agostiano, C. Bucci, L. Catucci, Toxicol Res (Camb) 2017, 6, 947-957; bG. Gopalakrishnan, C. Danelon, P. Izewska, M. Prummer, P. Y. Bolinger, I. Geissbuhler, D. Demurtas, J. Dubochet, H. Vogel, Angew Chem Int Ed Engl 2006, 45, 5478-5483; cX. Guo, Y. Zhang, J. Liu, X. Yang, J. Huang, L. Li, L. Wan, K. Wang, J Mater Chem B 2016, 4, 4191-4197.

[14] A. Lazzarini, A. Macchiarulo, A. Floridi, A. Coletti, S. Cataldi, M. Codini, R. Lazzarini, E. Bartoccini, G. Cascianelli, F. S. AmbesiImpiombato, T. Beccari, F. Curcio, E. Albi, Molecular Biology of the Cell 2015.

[15] K. C. Courtney, W. Pezeshkian, R. Raghupathy, C. Zhang, A. Darbyson, J. H. Ipsen, D. A. Ford, H. Khandelia, J. F. Presley, X. Zha, Cell Rep 2018, 24, 1037-1049.

[16] H. Zhang, Methods Mol Biol 2017, 1522, 1722.

[17] R. A. Schwendener, Ther Adv Vaccines 2014, 2, 159-182.

[18] aA. J. Metso, H. Zhao, I. Tuunainen, P. K. Kinnunen, Biochim Biophys Acta 2005, 1713, 83-91; bl. Reviakine, M. Gallego, D. Johannsmann, E. Tellechea, J Chem Phys 2012, 136, 084702; cJ. Kuntsche, J. C. Horst, H. Bunjes, Int J Pharm 2011, 417, 120-137.

[19] R. A. Schwendener, B. Ludewig, A. Cerny, O. Engler, in Liposomes: Methods and Protocols, Volume 1: Pharmaceutical Nanocarriers (Ed.: V. Weissig), Humana Press, Totowa, NJ, 2010, pp. 163-175. 
bioRxiv preprint doi: https://doi org/10.1101/2021.07.12.452073. this version posted July 13, 2021. The copyright holder for this preprint (which was not certified by peer review) is the author/funder, who has granted bioRxiv a license to display the preprint in perpetuity. It is made available under aCC-BY-NC 4.0 International license.

\section{Figures:}

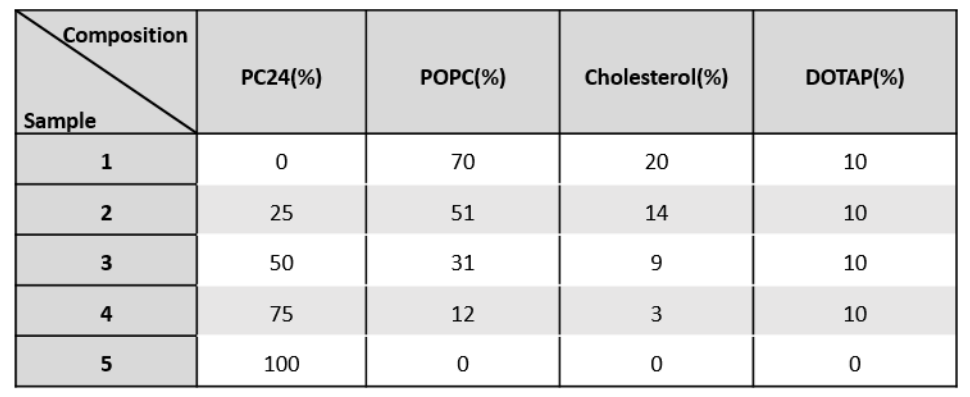

Table 1. Five different lipid compositions used for the NRs insertion studies with [PC24] increasing from $0 \%$ (sample 1) to $100 \%$ (sample 5).

\begin{tabular}{|l|c|c|c|}
\hline & $\begin{array}{c}\text { Membrane } \\
\text { Composition }\end{array}$ & $\begin{array}{c}\text { Native } \\
\text { membrane }\end{array}$ & $\begin{array}{c}\text { Thick } \\
\text { membrane }\end{array}$ \\
\hline POPC (\%) & 20 & 20 \\
\hline DOTAP (\%) & 10 & 10 \\
\hline PC24 (\%)
\end{tabular}

Table 2. Membrane components of SUV/NP preparations 
bioRxiv preprint doi: https://doi.org/10.1101/2021.07.12.452073; this version posted July 13, 2021. The copyright holder for this preprint (which was not certified by peer review) is the author/funder, who has granted bioRxiv a license to display the preprint in perpetuity. It is made available under aCC-BY-NC 4.0 International license.

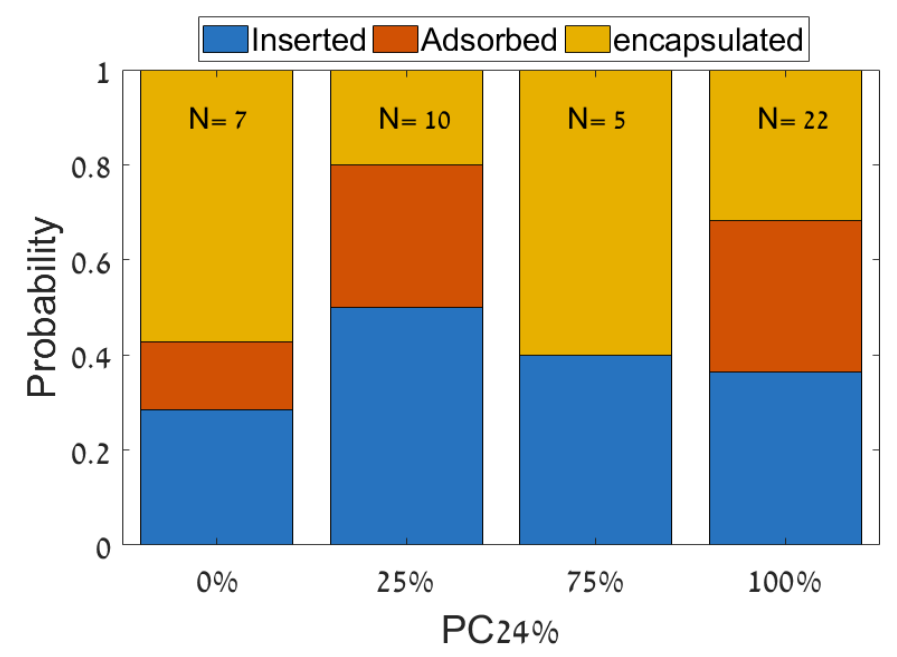

Figure 1: NRs membrane insertion efficiency as a function of PC24 lipid molar percentage in the SUVs' membrane composition. " the SUVs with 100\% PC24 composed a non round, distorted shaped vesicles, as shown in Figure S2. 
bioRxiv preprint doi: https://doi.org/10.1101/2021.07.12.452073: this version posted July 13, 2021. The copyriaht holder for this preprin (which was not certified by peer review) is the author/funder, who has granted bioRxiv a license to display the preprint in perpetuity. It is made available under aCC-BY-NC 4.0 International license.
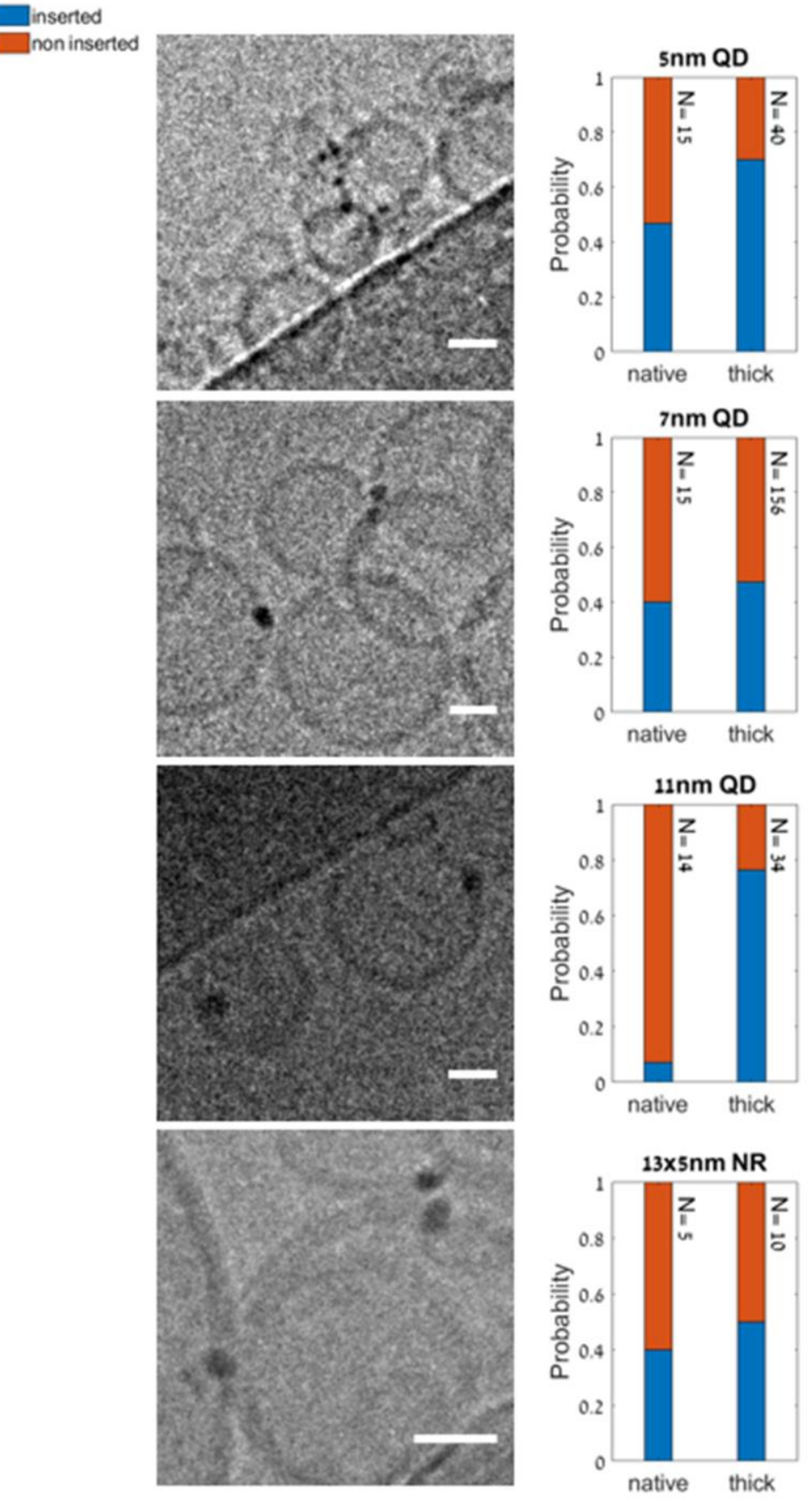

Figure 2: Incorporation of NPs into 'native' and 'thick' SUV membranes. Left column: cryoEM images showing examples for insertion of $5 \mathrm{~nm}, 7 \mathrm{~nm}, 11 \mathrm{~nm}$ QDs, and $5 \times 13 \mathrm{~nm}$ NRs into 'thick' SUV membranes. Right column: corresponding insertion efficiencies of 'native and 'thick' membranes. Lipid compositions for 'native' and 'thick' membranes are described in table 2 . Scale bars: $20 \mathrm{~nm}$. 
bioRxiv preprint doi: https://doi.org/10.1101/2021.07.12.452073; this version posted July 13, 2021. The copyright holder for this preprint (which was not certified by peer review) is the author/funder, who has granted bioRxiv a license to display the preprint in perpetuity. It is made available under aCC-BY-NC 4.0 International license.

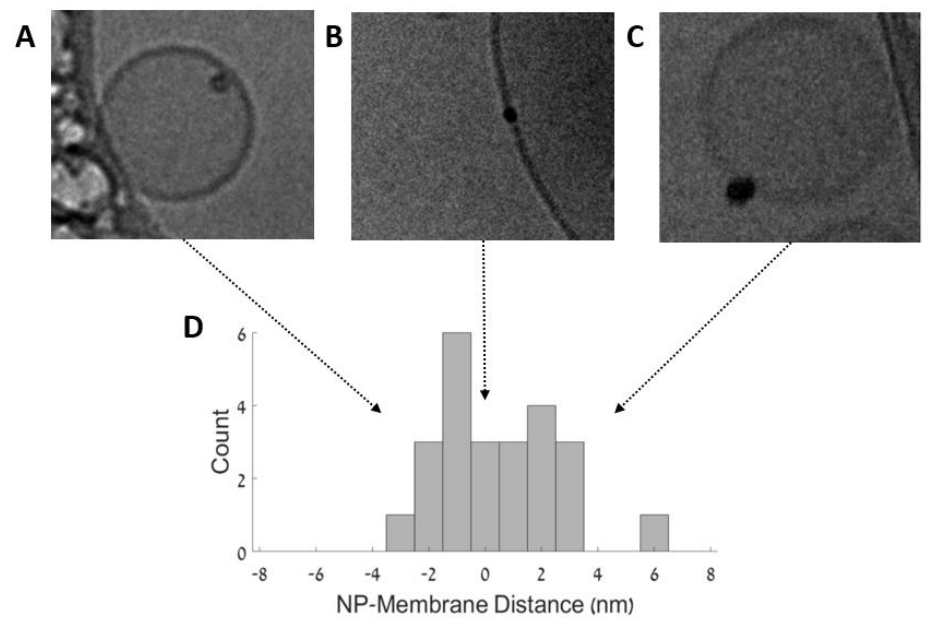

Figure 3: (a)-(c) Cryo-EM image representing QD inserted into thick SUVs (d) Position distribution of QDs interacting with thick SUVs lipid bilayer. Only QDs that were in the vicinity of a SUV, but not in the vicinity of other QDs or more than one SUV, were counted (24 QDs in total). 